\title{
Les déterminants de la demande en marques de distributeurs : étude à partir de deux produits de consommation courante
}

The characterics of private label demand: a study on two stapple goods

Fabian Bergès, Daniel Hassan et Sylvette Monier-Dilhan

\section{OpenEdition}

\section{Journals}

Édition électronique

URL : http://journals.openedition.org/economierurale/981

DOI : 10.4000/economierurale.981

ISSN : 2105-2581

Éditeur

Société Française d'Économie Rurale (SFER)

Édition imprimée

Date de publication : 15 novembre 2007

Pagination : 8-16

ISSN : 0013-0559

Référence électronique

Fabian Bergès, Daniel Hassan et Sylvette Monier-Dilhan, « Les déterminants de la demande en marques de distributeurs : étude à partir de deux produits de consommation courante ", Économie rurale [En ligne], 301 | Septembre-octobre 2007, mis en ligne le 15 novembre 2009, consulté le 21 avril 2019. URL : http://journals.openedition.org/economierurale/981 ; DOI : 10.4000/economierurale.981 


\title{
Les déterminants de la demande en marques de distributeurs : étude à partir de deux produits de consommation courante
}

\author{
Fabian BERGÈS, Daniel HASSAN, Sylvette MONIER-DILHAN • Toulouse School of Economics \\ (GREMAQ-INRA)
}

\section{Introduction}

e développement des Marques de distributeur (MDD) est l'un des phénomènes marquants de la grande distribution au cours des trois dernières décennies ${ }^{1}$. En France, dans le secteur alimentaire, la part en volume de ces produits représente aujourd'hui $25 \%$ des ventes de la grande distribution (40\% au Royaume-Uni et $20 \%$ au Etats-Unis) ${ }^{2}$. Ce phénomène a suscité une importante littérature économique, aussi bien théorique qu'empirique ${ }^{3}$. Toutefois, cette littérature est principalement dédiée à la compréhension de l'offre de MDD. Les principales questions abordées concernent en effet le partage du pouvoir de marché au sein de la structure verticale entre fabricants de Marques nationales (MN) et détaillants, le positionnement en qualité des MDD face aux MN, ou l'effet pro concurrentiel ou non de l'introduction des MDD. Tous ces travaux considèrent la demande de MDD comme donnée et ne s'intéressent pas aux facteurs expliquant l'achat des MDD.

Cette question a en revanche été abordée par la littérature marketing (Kapferer, 1998). Ainsi, Rao (1969) se demande s'il existe un segment délimité d'acheteurs de MDD ou bien si l'achat d'une marque d'enseigne ne traduit pas seulement un abandon impulsif

1. On date l'apparition des MDD avec la mise en linéaire des « produits libres » de Carrefour en 1976.

2. Voir Bergès-Sennou et Caprice, 2003.

3. Pour une bibliographie détaillée, voir Bergès-

Sennou et al, (2004). et momentané des $\mathrm{MN}$. Pour répondre à cette question, les travaux se sont développés dans deux directions. La première vise à appréhender les facteurs subjectifs qui influencent le choix du consommateur. La seconde direction tente d'établir un lien entre certaines caractéristiques objectives du consommateur et le type de marque achetée.

Les travaux liés à l'approche subjective s'accordent à souligner l'importance de la perception du consommateur. Les acheteurs attentifs à la composition des produits et sensibles au risque d'achat (ne pas acheter le produit le plus adéquat) achètent plus volontiers des MN que des MDD. Les consommateurs de MDD visent plutôt une qualité globalement satisfaisante, associée à un bon rapport qualité/prix (Livesey et Lennon, 1978 ; Baltas, 1997 ; Batra et Sinha, 2000). Ce ressort individuel se retrouve au niveau global. Batra et Sinha montrent en effet que les achats de MDD sont plus fréquents pour les biens de recherche (où l'information sur la qualité est disponible avant achat) que pour les biens d'expérience. Erdem et al. (2004) montrent également, à partir d'une étude internationale, que l'incertitude sur la qualité explique l'inégale attractivité des MDD pour le consommateur. Les caractéristiques extrinsèques-packaging, notoriété de la marque facteurs de réassurance du consommateur, figurent parmi les principaux atouts des MN (Richardson et al, 1994). De même, Sethuraman (2003) démontre que la disposition à payer un supplément de prix aux MN par rapport aux MDD n'est pas principalement liée à la perception d'un différentiel de qualité intrinsèque. 
L'approche objective des déterminants d'achat des MDD distingue deux types de caractéristiques du consommateur pouvant influer sur son choix de marque. Les premières concernent l'identité de l'acheteur (âge, revenu, habitat...), les secondes portent sur son comportement d'achat. Sur ce second point, les travaux, relativement récents, aboutissent à des résultats convergents. Bonfrer et Chintagunta (2004) étudient la relation entre la fidélité (aux marques et aux enseignes) et le choix MN ou MDD et établissent que la fidélité à l'enseigne augmente la probabilité d'achat des MDD tandis que la fidélité à la marque la réduit. Sur ce dernier point (fidélité à la marque), leur résultat rejoint celui de Baltas (op. cit.). Par ailleurs, différentes recherches montrent que les promotions ont un impact plus fort sur l'achat des MN que sur celui des MDD (Bronnenberg et Wathieu, 1996).

En revanche, les recherches sur l'impact des facteurs sociodémographiques ne débouchent pas sur un consensus. Les premiers travaux empiriques pour les États-Unis montrent que les variables démographiques sont de médiocres prédicteurs du choix des marques (Frank et Boyd, 1965 ; Burger et Schott, 1972 ; Livesey et Lennon, op. cit.) Même le revenu, dont le pouvoir explicatif attendu était fort, puisque les MDD sont sensiblement moins chères que les $\mathrm{MN}$, paraît ne jouer qu'un rôle secondaire. Les acheteurs de MDD ne constituent pas un segment particulier de consommateurs. Des travaux plus récents aboutissent toutefois à des résultats moins tranchés. Selon Richardson et al. (1996), le revenu et la taille de la famille influent sur l'achat des MDD (négativement dans un cas, positivement dans l'autre). De même, Dhar et Hoch (1997) montrent que la part de marché des MDD augmente dans les zones où la population est plus âgée ou moins fortunée. Enfin, Cole et Sethuraman (1999) concluent que la disposition à payer pour les MN est forte à la fois chez les hauts et les bas revenus et faible dans les tranches moyennes.

Ces résultats mitigés militent pour un réexamen du problème. Une seconde raison qui incite à un tel réexamen tient à la nature « déclarative » des données sur lesquelles reposent de nombreux travaux portant sur cette question. Or il existe potentiellement un biais entre intentions d'achats et achats effectifs. Si les données déclaratives sont difficilement contournables lorsqu'il s'agit d'appréhender la perception du consommateur, des données issues « d'achats effectifs » sont mieux adaptées à l'étude du lien entre choix de marques et caractéristiques objectives des acheteurs. Certains travaux cités reposent sur des données individuelles d'achats constatés mais les panels sont peu importants (quelques centaines d'observations). Grâce à des données nombreuses et détaillées concernant la France, nous revisitons cette question de l'impact des caractéristiques en combinant les aspects socio-économiques et ceux liés au comportement d'achat. La richesse des données permet également d'intégrer dans l'analyse des variables qui tiennent compte de la différenciation des produits. En effet, la concurrence entre $\mathrm{MN}$ et MDD est, pour un même produit, sensible au créneau considéré. Il est donc important de contrôler cet effet variété. De même, les politiques d'enseigne ayant un impact sur l'attractivité des MDD, nous prenons en compte ce facteur.

La première partie présente les données et le modèle. Les résultats font l'objet de la deuxième partie. La conclusion met en perspective l'originalité de cette étude face aux résultats obtenus.

\section{Les données et le modèle}

Nous travaillons sur des données extraites du panel représentatif de consommateurs Sécodip (Société d'études de la consommation, de la distribution et de la publicité) pour l'année 2000. Nous disposons, d'une part d'informations concernant les achats 
réalisés par un panel de 8000 ménages répartis sur tout le territoire français pour leur consommation à domicile (date d'achat, marque, poids et prix du produit, enseigne où l'achat a eu lieu, etc.) et, d'autre part, de renseignements sur des caractéristiques socio-économiques de ces ménages (revenu, niveau d'étude, lieu d'habitation, etc.).

Nous observons le choix des ménages concernant la marque des produits et étudions à partir de ce panel les déterminants du choix du type de marque (MN ou MDD) pour deux produits de grande consommation : les pâtes alimentaires et les yaourts. Pour être sûrs que les consommateurs font face à un vrai choix entre MN et MDD, nous centrons l'étude sur le cœur de marché pour chaque produit, c'est-à-dire les yaourts au lait de vache en pots plastique et les pâtes alimentaires standard de cuisson normale. Nous ne retenons pas certains produits spécifiques vendus principalement sous MN (yaourts au lait de brebis, pâtes fantaisies, etc.). En effet, pour l'année 2000, les produits MDD haut de gamme ont, en pâtes et yaourt, des parts de marché négligeables. Pour chaque type de marque, le consommateur peut acheter un produit sous MN ou un proche substitut sous MDD (produits $m e-t o o)^{4}$. Ces produits sont caractérisés par des achats fréquents (73 997 pour les yaourts et 27315 pour les pâtes) réalisés par environ 7000 ménages. La part de marché des MDD est légèrement supérieure à la moyenne pour les produits alimentaires qui est de $25 \%$ (tableau 1).

Sur les linéaires des Grandes et moyennes surfaces (GMS), on trouve outre les MN et les MDD, des produits «premiers prix », vendus pour concurrencer les produits Hard Discount. Toutefois, la part de marché des premiers prix en pâtes et en yaourts est faible : $3,5 \%$ et $4 \%$ respectivement. Cela permet de ne pas tenir compte de ce troisième élément du choix des consomma-

4. Ces produits sont destinés à concurrencer ceux sous marques nationales. teurs. L'étude n'est par ailleurs pas pénalisée : car le modèle utilisé est un logit binomial car nous nous intéressons au choix dichotomique du ménage face aux MN et aux MDD. Le tableau 1 présente quelques statistiques sur ces produits.

Pour les deux produits, on observe le constat classique selon lequel le prix des MDD est inférieur à celui des MN. Cette différence est forte $(34 \%)$ pour les yaourts, elle n'est que de $9 \%$ en moyenne pour les pâtes.

Les modèles de choix discrets sont adaptés à l'étude du choix des consommateurs entre les deux types de marques. Le logit multinomial s'applique aux cas où les variables explicatives sont spécifiques aux individus ${ }^{5}$. L'idée sur laquelle ce modèle est construit est présentée dans l'encadré 1 .

Les statistiques descriptives concernant les variables explicatives $\left(x_{i}\right)$ utilisées dans la régression sont exposées dans le tableau 1. Ces variables sont les suivantes: 1. Des variables socio-économiques (première partie du tableau 1) : âge, lieu d'habitation, diplôme et revenu du ménage. Pour le lieu d'habitation, nous avons distingué les grandes agglomérations (de plus de 200000 habitants) des autres. En effet, le commerce traditionnel étant plus présent dans ces agglomérations et les MDD étant essentiellement distribuées en GMS, le signe attendu de la variable «grande agglomération » est négatif. Pour le diplôme, nous tenons compte du niveau d'éducation du chef de famille en distinguant selon que le diplôme est supérieur ou non au niveau bac +2 ans. Pour le revenu, nous considérons quatre tranches : revenu mensuel inférieur à $762 €$, revenu compris entre $762 €$ et $1524 €$, revenu compris entre $1524 €$ et $3048 €$ et revenu supérieur à $3048 €$.

5. Lorsque les variables, ou un sous-ensemble, sont propres aux alternatives le modèle de choix adapté est le logit conditionnel. 


\section{Encadré 1. Le modèle logit binomial}

La probabilité que le ménage i achète la marque $\mathrm{M}$ dépend de ses caractéristiques $x_{i}$. Les paramètres $\beta_{M}, M=M N$ ou $M D D$ varient selon les modalités. La probabilité que le ménage dont les caractéristiques sont $x_{i}$ achète la marque $i$ s'écrit donc :

$$
P\left(M / x_{i}\right)=\frac{\exp \left(\beta_{M}^{\prime} x_{i}\right)}{\exp \left(\beta_{M N}^{\prime} x_{i}\right)+\exp \left(\beta_{M D D}^{\prime} x_{i}\right)}
$$

Pour résoudre le problème d'identification du modèle, nous imposons la nullité des coefficients associés au choix de la marque MN, qui est l'alternative de référence. La probabilité d'acheter une MDD s'écrit alors :

$$
P\left(M D D / x_{i}\right)=\frac{\exp \left(\beta_{M D D}^{\prime} x_{i}\right)}{1+\exp \left(\beta_{M D D}^{\prime} x_{i}\right)}
$$

Les coefficients $\beta_{M D D}$ ne renseignent que sur le sens d'évolution de la probabilité d'acheter une MDD mais pas sur son amplitude. Nous calculerons donc pour chaque variable l'effet marginal d'un changement de modalité (pour une variable dichotomique) ou d'unité (pour une variable continue).

\section{Des variables décrivant les habitudes} d'achat (deuxième partie du tableau 1) : quantité de produit achetée lors de chaque achat, nombre d'enseignes annuellement visitées, nombre de marques achetées au cours de l'année, écart-type du prix des achats mesurant le caractère plus ou moins captif du consommateur par rapport au prix. Ces variables sont propres à chaque consommateur.

- La quantité de produit achetée lors de chaque achat devrait avoir un effet positif sur la probabilité d'acheter une MDD, afin de limiter la dépense.

- Le nombre d'enseignes visitées et le nombre de marques achetées par un ménage ${ }^{6}$ sont mesurés par l'inverse de l'indice d'Herfindahl. L'inverse de cet indice indique le nombre équivalent d'enseignes visitées ou de marques achetées par le ménage. Un indice élevé traduit une forte concentration à laquelle correspond un petit nombre d'enseignes ou de marques. Cet indice est calculé pour chaque ménage, à partir de ses parts de dépense par enseigne ou par marque selon le $\operatorname{cas}^{7}$. Utiliser cet indice permet de pondérer le nombre d'enseignes visitées ou de marques achetées par l'importance des achats du ménage. Ces nombres constituent des indicateurs de la fidélité du consommateur à l'enseigne et à la marque ${ }^{8}$. Une mesure alternative de cette fidélité est obtenue en considérant la part maximale de dépense respectivement par enseigne et par marque. Ces mesures étant corrélées, seuls les indicateurs dérivés des indices d'Herfindahl ont été retenus comme variables explicatives.

- L'écart-type : les consommateurs achè-

6. Les MDD sont traitées comme une seule et même marque quelle que soit l'enseigne dans laquelle elles ont été achetées. Par ailleurs, l'étude portant sur l'année 2000, les marques de distributeur dites « haut de gamme »n'existaient pas encore : une seule MDD (produit me-too) était en effet disponible dans chaque enseigne.

7. Cet indice est égal à $\sum_{j=1}^{J}\left(\frac{\text { valeur annuelle des achats du ménage dans la marque } j}{\text { valeur annuelle des achats du ménage pour le produit }}\right)^{2}$, avec $j=\{M D D, M N 1, M N 2, \ldots\}$

8. Voir par exemple les apports de la littérature en Marketing sur les indices de fidélité tels que l'article fondateur de Cunningham (1961), ou l'ouvrage de Roberston et Kassarjian (1991) sur le comportement du consommateur. 
Tableau 1. Statistiques descriptives sur les ménages et les achats

\begin{tabular}{l|c|c|c|c}
\hline Produit & Yaourt & \multicolumn{2}{|c}{ Pâtes } \\
\hline Nombre d'actes d'achat & \multicolumn{2}{|c|}{73997} & \multicolumn{2}{|c}{27315} \\
\hline Part de marché des MDD (en valeur) & \multicolumn{2}{|c|}{$27 \%$} & \multicolumn{2}{|c}{$31 \%$} \\
\hline Prix moyen au kilo (en $€$ ) & \multicolumn{2}{|c|}{1,82} & \multicolumn{2}{|c}{1,79} \\
\hline Prix moyen au kilo (en $€$ ) & MN & MDD & MN & MDD \\
& 2,04 & 1,36 & 1,86 & 1,68 \\
\hline
\end{tabular}

\begin{tabular}{|c|c|c|}
\hline \multicolumn{3}{|c|}{ 1. Variables sociodémographiques et économiques des ménages } \\
\hline Âge & \multicolumn{2}{|c|}{46 ans } \\
\hline Habite dans une agglomération de plus de 200000 habitants & \multicolumn{2}{|c|}{$32 \%$} \\
\hline Études supérieures (diplôme $\geq$ Bac +2 ) & \multicolumn{2}{|c|}{$17 \%$} \\
\hline \multicolumn{3}{|l|}{ Revenu du ménage } \\
\hline Revenu mensuel $<762 €$ & \multicolumn{2}{|c|}{$4 \%$} \\
\hline $762 € \leq$ Revenu mensuel $<1524 €$ & \multicolumn{2}{|c|}{$26 \%$} \\
\hline $1524 € \leq$ Revenu mensuel $<3048 €$ & \multicolumn{2}{|c|}{$55 \%$} \\
\hline Revenu mensuel $\geq 3048 €$ & \multicolumn{2}{|c|}{$15 \%$} \\
\hline \multicolumn{3}{|c|}{ 2. Variables de comportement des ménages } \\
\hline Quantité achetée lors de l'achat (kg) - Moyenne et écart-type & $1,33(0,62)$ & $0,61(0,32)$ \\
\hline Nombre d'enseignes visitées dans l'année - Moyenne et écart-type & \multicolumn{2}{|c|}{$2,02(0,82)$} \\
\hline \% maximum de dépense dans une même enseigne & \multicolumn{2}{|c|}{$66,1 \%$} \\
\hline Nombre de marques achetées dans l'année & 2,76 & 2,59 \\
\hline$\%$ maximum de dépense dans une même marque & $56,8 \%$ & $59,6 \%$ \\
\hline Ecart type des prix d'achat $(€)$ & 0,45 & 0,54 \\
\hline \multicolumn{3}{|l|}{ 3. Caractéristiques du produit } \\
\hline Promotion remarquée par le ménage & $8,8 \%$ & $5,1 \%$ \\
\hline Yaourt sucré & $30 \%$ & /I \\
\hline Yaourt entier & $27,7 \%$ & /I \\
\hline Yaourt maigre & $32,7 \%$ & /I \\
\hline Yaourt aromatisé & $67,4 \%$ & /I \\
\hline Pack de 4 yaourts & $29,7 \%$ & /I \\
\hline Pack de 12 yaourts & $35 \%$ & /I \\
\hline Pack de 16 yaourts & $35,3 \%$ & /I \\
\hline Pâtes vendues en boîte & /I & $29 \%$ \\
\hline Pâtes vendues en sachet & /I & $71 \%$ \\
\hline Pâtes de qualité standard & /I & $25 \%$ \\
\hline Pâtes de qualité supérieure & /I & $75 \%$ \\
\hline
\end{tabular}

tent dans des intervalles de prix plus ou moins larges. Certains font leurs achats dans une gamme de prix réduite, d'autres, au contraire, expérimentent davantage. Ces attitudes sont reflétées par l'écart-type du prix des achats réalisés par le ménage au cours de l'année 2000. Nous observons dans quelle mesure de telles attitudes influencent le choix du type de marque.
3. Les variables caractéristiques du produit (troisième partie du tableau 1) : la variable promotion indique si le produit bénéficiait d'une promotion lorsque le ménage l'a acheté. La prise en compte de cette variable permet de contrôler l'effet promotion. Les promotions se répartissent de la façon suivante : 5,85\% des achats de pâtes sont réalisés en promotion pour les 
$\mathrm{MN}$ et $4 \%$ pour les MDD ; en yaourt ces pourcentages sont respectivement 9,56 et $7,34^{9}$. Par ailleurs, il s'agit de savoir si certaines caractéristiques sont favorables à un type de marque : type d'emballage (sachet versus boîte pour les pâtes), nombre d'articles par lot, composition. Nous capturons également l'impact des politiques d'enseigne concernant les MDD par un effet fixe propre à chacun des six principaux distributeurs ${ }^{10}$. Ces enseignes en 2000 représentaient $80 \%$ des ventes des GMS en produits de grande consommation.

Un tiers des ménages habitent dans une grande agglomération. Le revenu moyen par ménage et le pourcentage de chefs de famille ayant un diplôme supérieur à bac + 2 ans sont proches de ceux de la population totale $^{11}$. Au cours de l'année 2000, chaque ménage visite 2 enseignes et effectue $66 \%$ de ses dépenses dans une même enseigne ${ }^{12}$. Le nombre de marques achetées est en moyenne de 2,6 par an, mais le ménage concentre plus de la moitié de ses dépenses sur une seule marque.

\section{Les résultats}

Pour les deux produits, l'estimation est satisfaisante : le taux de prédiction correcte est égal à $74 \%$ et le pseudo-R ${ }^{2}$ de $\mathrm{McFadden}$ est supérieur à 0,20 (cf. tableau 2). Par ailleurs, la très grande majorité des coefficients est significative pour un niveau d'erreur inférieur $1 \%$ (cf.tableau 3). La probabilité moyenne d'achat de MDD estimée est de $33 \%$ pour les yaourts et de $39 \%$ pour les pâtes.

9. Un test de moyenne montre que ces chiffres sont significativement différents entre MN et MDD pour chacun des produits.

10. Les six principaux distributeurs considérés sont : Auchan, Carrefour, Casino, Intermarché, Leclerc et Système U.

11. Cf. INSEE (2000), La France en faits et chiffres. 12. De tels chiffres sont dans le même ordre de grandeur que l'enquête effectuée par Chardon et Dumartin (1998).
Tableau 2. Caractéristiques et qualité du modèle logistique

\begin{tabular}{l|c|c}
\hline Produit & Yaourt & $\begin{array}{c}\text { Pâtes } \\
\text { alimentaires }\end{array}$ \\
\hline Nombre d'observations & 73997 & 27315 \\
\hline Pseudo R & 0,21 & 0,23 \\
\hline Prévisions correctes & $74 \%$ & $74 \%$ \\
\hline Sensibilité* & $48 \%$ & $59 \%$ \\
\hline Précision $^{* *}$ & $87 \%$ & $83 \%$ \\
\hline $\begin{array}{l}\text { Probabilité moyenne } \\
\text { d'achat MDD }\end{array}$ & $32,7 \%$ & $38,6 \%$ \\
\hline
\end{tabular}

* La sensibilité du modèle correspond au nombre de prévisions correctement estimées d'achat de MDD sur le nombre d'achats effectifs de MDD.

** La précision du modèle correspond au nombre de prévisions correctement estimées d'achat de MN sur le nombre d'achats effectifs de MN.

L'influence de chaque caractéristique est appréciée par son effet marginal. Celui-ci mesure comment varie la probabilité d'achat d'une MDD lorsque la modalité de la variable change d'une unité et, pour les variables dichotomiques, selon que le caractère est présent ou non. Ces effets marginaux sont calculés selon la méthode proposée par Chamberlain (1982). Cette méthode permet de s'affranchir d'un individu moyen imaginaire pour l'interprétation des résultats, car l'effet marginal est calculé sur tout le sous échantillon concerné ${ }^{13}$.

13. Avec la méthode de Chamberlain, pour évaluer l'effet marginal d'une variable dichotomique, on considère le sous-échantillon pour lequel cette variable vaut 0 . On prédit, pour chaque observation, la probabilité d'achat de MDD en imposant que cette variable prenne la valeur 1 . L'effet marginal de la variable considérée est alors l'effet marginal moyen calculé sur le sous-échantillon. Pour la variable ordonnée revenu, nous évaluons l'effet marginal en termes d'enrichissement : de «pauvre à modeste », de « modeste à aisé » et enfin de « aisé à riche ». Par exemple, pour l'effet marginal de " modeste à aisé », nous ne prenons en compte que les individus de la catégorie «modeste » et pas ceux des catégories « pauvre» ou « riche». 
Nous présentons les résultats du modèle (tableau 3) en examinant successivement les trois types de variables explicatives prises en compte pour la régression. Le référentiel choisi dans la modélisation logistique étant la modalité MN, les paramètres traduisent comment varie la probabilité d'acheter une MDD plutôt qu'une MN suite à la modification d'une caractéristique.

\section{Caractéristiques socio-économiques}

L'âge et le diplôme n'ont pas d'influence sur le choix de la marque. Ce résultat ne dépend pas de la manière dont ces variables ont été prises en compte ${ }^{14}$ (découpage et définition des classes).

14. L'âge a aussi été introduit sous la forme de classes d'âge et différents découpages de classes ont été envisagés pour le diplôme.

Tableau 3. Déterminants de la probabilité d'acheter une MDD plutôt qu'une MN (Effets marginaux exprimés en pourcentage)

\begin{tabular}{|c|c|c|c|}
\hline \multicolumn{2}{|l|}{ Variables } & Yaourt & Pâtes \\
\hline \multicolumn{4}{|c|}{ 1. Caractéristiques socio-économiques } \\
\hline \multicolumn{2}{|l|}{ Âge du panéliste } & $-0,09$ & n.s. \\
\hline \multicolumn{2}{|l|}{ Habite dans une ville $>200000$ hab. } & $-2,77$ & $-3,06$ \\
\hline $762 € \leq$ Revenu mensuel $<1524 €$ & \multirow{3}{*}{$\begin{array}{l}\text { Le revenu mensuel } \\
\text { de référence est inférieur } \\
\text { à } 762 € \text { mensuels }\end{array}$} & $-6,16$ & $-7,31$ \\
\hline $1524 € \leq$ Revenu mensuel $<3048 €$ & & $-2,65$ & $-1,77$ \\
\hline Revenu mensuel $\geq 3048 €$ & & $-3,47$ & $-1,73$ \\
\hline \multicolumn{2}{|l|}{ Diplômé du supérieur $(\geq \mathrm{Bac}+2)$} & n. s. & n. s. \\
\hline \multicolumn{4}{|c|}{ 2. Comportement d'achat } \\
\hline \multicolumn{2}{|c|}{ Quantité achetée lors de l'acte d'achat } & 5,13 & 11,42 \\
\hline \multicolumn{2}{|l|}{ Nombre d'enseignes (indice pondéré) } & $-4,74$ & $-7,26$ \\
\hline \multicolumn{2}{|l|}{ Nombre de marques (indice pondéré) } & 8,79 & 9,68 \\
\hline \multicolumn{2}{|l|}{ Ecart-type des prix d'achats } & 13,51 & 10,62 \\
\hline \multicolumn{4}{|c|}{ 3. Caractéristiques du produit } \\
\hline \multicolumn{2}{|c|}{ Promotion remarquée par le ménage } & $-6,03$ & $-11,31$ \\
\hline \multicolumn{2}{|l|}{ Yaourt sucré (par rapport à non sucré) } & $-15,37$ & // \\
\hline Yaourt au lait entier & \multirow{3}{*}{$\begin{array}{c}\text { La référence est } \\
\text { le yaourt au lait normal }\end{array}$} & n.s. & $/ 1$ \\
\hline Yaourt au lait maigre & & $-16,73$ & /I \\
\hline Yaourt aromatisé (par rapport à nature) & & $-16,03$ & /I \\
\hline Pack de 4 yaourts & \multirow{2}{*}{$\begin{array}{c}\text { La référence est } \\
\text { le pack de } 16 \text { yaourts }\end{array}$} & n.s. & /I \\
\hline Pack de 12 yaourts & & 14,97 & /I \\
\hline \multicolumn{2}{|c|}{ Pâtes vendues en boîte (par rapport à « en sachet ») } & /I & $-42,64$ \\
\hline \multicolumn{2}{|c|}{ Pâtes qualité supérieure (par rapport à standard) } & $/ /$ & $-26,80$ \\
\hline Enseigne 1 & \multirow{6}{*}{$\begin{array}{c}\text { Par rapport à } \\
\text { « autres enseignes » }\end{array}$} & 15,86 & 4,98 \\
\hline Enseigne 2 & & $-5,58$ & 5,58 \\
\hline Enseigne 3 & & 3,20 & n.s. \\
\hline Enseigne 4 & & $-1,93$ & 11,36 \\
\hline Enseigne 5 & & n.s. & 9,23 \\
\hline Enseigne 6 & & $-0,95$ & $-3,33$ \\
\hline
\end{tabular}

n.s. $=$ non significativement différents de 0 pour un niveau d'erreur supérieur à $10 \%$.

Tous les autres coefficients sont significatifs pour un niveau d'erreur inférieur à $1 \%$. 
Soulignons la convergence des résultats pour les deux produits étudiés.La taille de l'agglomération joue, mais très faiblement : la probabilité de choisir une MDD décroît de $3 \%$ lorsque le ménage habite dans une grande ville. Enfin, il existe un effet revenu mais son impact est surtout marqué pour les bas revenus : passer de «pauvre » à « modeste » décroît la probabilité d'acheter une MDD de $7 \%$ environ. Il diminue pour les catégories à revenu supérieur.

\section{Comportement d'achat}

Lorsque la quantité de produit achetée augmente, la probabilité d'acheter une MDD est plus forte. Ce qui traduit un comportement de limitation de la dépense, le prix des MDD étant en moyenne inférieur à celui des MN.

La fidélité à l'enseigne est l'un des déterminants du comportement d'achat face aux MDD. Lorsqu'elle augmente (i.e. lorsque le nombre d'enseignes visitées dans l'année décroît), le consommateur achète plus de MDD ( $+5 \%$ en yaourt et $+7 \%$ en pâtes lorsque le nombre d'enseignes visitées décroît d'une unité). À l'inverse, l'attachement aux marques (d'autant plus fort que le nombre de marques achetées est faible) n'est pas favorable aux MDD : la probabilité d'achat des MDD diminue d'environ $9 \%$ dès lors que le ménage réduit son éventail de marques d'une unité.

Par ailleurs, le choix MDD est davantage le fait de ménages qui achètent dans un éventail de prix étroit. En effet si l'écart type des achats du ménage diminue d'un euro, la probabilité d'achat d'une MDD augmente de $13,5 \%$ pour le yaourt et de $10,6 \%$ pour les pâtes. Les « acheteurs de MDD » expérimentent donc peu en termes de prix.

\section{Caractéristiques du produit}

L'introduction de ces variables permet de capturer des caractéristiques qui sont plus spécifiques au segment de marché $\mathrm{MN}$ ou alternativement MDD.
L'impact promotionnel sur le choix du type de marque est défavorable aux MDD : diminution de la probabilité d'acheter une MDD de 6 à $11 \%$ en présence d'une promotion remarquée par le ménage.

Nous nous intéressons au cœur de marché ( $c f$. la première partie «Les données et le modèle »), mais l'homogénéité des produits n'est pas parfaite : le conditionnement, le caractère sucré, aromatisé, etc. maintiennent une différenciation des biens. L'achat de pâtes conditionnées en boîte ou portant la mention «qualité supérieure » s'accompagne d'une diminution de la probabilité d'achat des marques propres. En yaourt, les consommateurs sont davantage enclins à se diriger vers des $\mathrm{MN}$ dès lors qu'ils désirent certaines caractéristiques sortant du strict cœur de marché ${ }^{15}$ : sucré, au lait maigre ou aromatisé. Sur tous ces différents segments, des MDD sont pourtant disponibles.

Chaque enseigne ayant développé sa politique propre de communication ainsi que ses MDD, toutes n'ont pas la même influence sur le consommateur. L'enseigne E1 (cf. tableau 3) se démarque nettement avec un fort impact sur l'achat des MDD, et ce pour les deux produits. Pour les pâtes alimentaires, les enseignes 4 et 5 bénéficient de leur expérience : elles ont été parmi les premières à avoir investi dans la communication sur leurs MDD.

\section{Conclusion}

Les résultats obtenus permettent de saisir certains déterminants d'achat des MDD pour les produits de grande consommation.

Nous montrons d'abord que les variables sociodémographiques ne sont pas influentes. Seul un effet revenu peut être mis en évidence, mais son amplitude est modeste. Ces résultats vont dans le sens des premiers

15. Bontemps et al. (2004) montrent qu'une stratégie des MN, face au développement des MDD, consiste à se recentrer sur des produits plus élaborés. 
travaux empiriques selon lesquels l'identité du consommateur explique mal le choix de la marque.

En revanche, notre travail confirme que le choix MDD versus MN est lié à des caractéristiques du comportement d'achat. Comme l'ont déjà montré Bonfrer et Chintagunta (2004) pour les États-Unis, la fidélité à l'enseigne favorise le choix MDD. Les politiques de fidélisation aux enseignes (cartes de fidélité, coupons, ...) ont donc un effet sur l'achat des MDD. Au contraire, les acheteurs de MDD se recrutent parmi les consommateurs peu fidèles aux marques.

Ensuite, cette recherche met en évidence l'impact des caractéristiques propres au produit. Dès que l'on sort du strict cœur de marché (recettes nouvelles, conditionnement...), la demande s'oriente davantage vers les MN. Celles-ci bénéficient en effet d'une plus forte capacité d'innovation en termes de « recette » ou de packaging.
Lorsque les MDD adoptent ces nouveaux produits, elles ne comblent pas instantanément leur retard sur les $\mathrm{MN}$ en termes d'image sur ces segments.

Enfin, les prolongements à donner à cette étude sont directement liés à l'évolution de la grande distribution. En effet, les MDD retenues ici constituent un segment particulier des marques d'enseigne : les produits me-too, caractérisés par leur proximité avec les MN standard. Il convient de prendre en compte les MDD récemment introduites qui se situent explicitement sur le créneau de la haute qualité. Par ailleurs, la grande distribution est aujourd'hui confrontée à la concurrence des "hard discounters ». L'étude du choix de marque devra intégrer ces nouveaux produits.

Les auteurs remercient Christophe Bontemps (Inra) pour son aide dans le traitement des données ainsi que Grégory Verdugo pour son appui en informatique. 


\section{RÉFÉRENCES BIBLIOGRAPHIQUES}

Batra Rajeev, Sinha Indrajit (2000). Consumer-Level Factors Moderating the Success of Private Label Brands across Product Categories. Journal of Retailing, 76 (2), p. 175-191.

Baltas G. (1997). Determinants of Store Brand Choice: a Behavioral Analysis. Journal of Product and Brand Management, 6(5), p. 315-324.

Bergès-Sennou F., Bontems Ph., Réquillart V. (2004). Economics of Private Labels: A Survey of Literature. Journal of Agricultural and Food Industrial Organization, 2(3), 25 pages, www.bepress.com/jafio

Bergès-Sennou F., Caprice S. (2003). Les rapports producteurs-distributeurs : Fondements et implications de la puissance d'achat. Économie Rurale, $\mathrm{n}^{\circ}$ 277-278, p.192-205.

Bonfrer A., Chintagunta P. (2004). Store Brands: Who Buys Them and What Happens to Retail Prices When They Are Introduced? Review of Industrial Organization, vol. 24, p. 195-218.

Bontemps C., Orozco V., Réquillart V., Trévisiol A. (2004). Price Effects of Private Label Development. In Journal of Agricultural and Food Industrial Organization, 19 pages, à paraître, www.bepress.com/jafio

Bronnenberg B., Wathieu L. (1996). Asymmetric Promotion Effects and Brand Positioning. Marketing Science, vol 15, $\mathrm{n}^{\circ} 4$.

Burger Ph., Schott B. (1972). Can Private Brand Buyers Be Identified? Journal of Marketing Research, 9 (May), p. 219-222.

Chamberlain G. (1982). Multivariate Regression Models for Panel Data. Journal of Econometrics, vol. 18, p. 5 - 46.

Chardon O., Dumartin S. (1998). Fidélité aux enseignes, fidélité aux marques : le choix des consommateurs. INSEE Première, $\mathrm{n}^{\circ} 609$, disponible sur www.insee.fr
Cole C., Sethuraman R. (1999). Factors Influencing the Price Premiums that Consumers Pay for National Brands over Store Brand. Journal of Product and Brand Management, vol 8(4), p. 340-351.

Cunningham R.-M. (1961). Customer Loyalty to Store and Brand. Harvard Business Review, vol. 39, p. 127 - 137.

Dhar Sanjay K., Hoch S.-J. (1997). Why Store Brand Penetration Varies by Retailer? Marketing Science, 16(3), p. 208 - 227.

Erdem Tülin, Zhao Ying, Valenzuela A. (2004). Performance of Store Brands: a Cross-Country Analysis of Consumer Store Brand Preferences, Perceptions and Risk. Journal of Marketing Research, vol. XLI, p. 86-100.

Frank R.-E., Boyd H.-W. (1965). Are PrivateBrand Prone Grocery Customers Really Different? Journal of Marketing Research, 2(4), p. 27-35.

Kapferer J.-N. (1998) Les marques. Editions Organisation ;

Livesey F., Lennon P. (1978). Factors Affecting Consumers' Choice. European Journal of Marketing, 12(2), p.158 - 170.

Rao T. (1969). Are Some Consumers More Prone to Purchase Private Brands? Journal of Marketing Research, vol. VI, november, p. 447-450.

Richardson P., Jain Arun (1996). Household Store Brand Proneness: A Framework. Journal of Retailing, Summer, 72(2), p. 159-186.

Richardson P., Dick A., Jain Arun (1994). Extrinsic and Intrinsic Cue Effects on Perceptions of Store Brand Quality. Journal of Marketing, 58, october, p. 28-36.

Roberston Th., Kassarjian H. (1991). Handbook of Consumer Behavior. Prenctice Hall College, 614 p.

Sethuraman R. (2003). Measuring National Brands Equity over Store Brands and Exploring its Antecedents. Marketing Science, september-october, $28 \mathrm{p}$. 